\title{
Ecocardiografía Doppler en conejos neozelandeses blancos normales*
}

\author{
Pidal, G. ${ }^{1}$; D’Anna, E. ${ }^{2}$; Pérez Valega, E. ${ }^{3}$; Lightowler, C. ${ }^{1}$ \\ ${ }^{1}$ Instituto de Fisiopatología Cardiovascular, ${ }^{2}$ Unidad de Diagnóstico por Imágenes, Hospital Escuela, Facultad de \\ Ciencias Veterinarias, UBA, Avda. Chorroarin 280 (1427), Buenos Aires, Argentina. E-mail: gapidal@yahoo.com.ar. \\ ${ }^{3}$ Cátedra de Semiología, Facultad de Ciencias Veterinarias, UNNE, Corrientes, Argentina.
}

\begin{abstract}
Resumen
Pidal, G.; D’Anna, E.; Pérez Valega, E.; Lightowler, C.: Ecocardiografía Doppler en conejos neozelandeses blancos normales. Rev. vet. 21: 2, 106-111, 2010. Los autores establecen las ventanas ecocardiográficas para el registro Doppler en conejos neozelandeses blancos normales. Asimismo describen las características de los distintos flujos transvalvulares y determinan valores normales para el tiempo de relajación isovolumétrico (TRIV) del ventrículo izquierdo, la velocidad pico (Vp) y tiempo de eyeccion (Tey) ventricular para las válvulas sigmoideas. Para los flujos atrioventriculares establecen la velocidad pico para las ondas E y A y la relación E/A para el flujo transmitral.
\end{abstract}

Palabras clave: conejo, ecocardiografía Doppler, flujos transvalvulares.

\begin{abstract}
Pidal, G.; D'Anna, E.; Pérez Valega, E.; Lightowler, C.: Doppler echocardiography in normal New Zealand white rabbits. Rev. vet. 21: 2, 106-111, 2010. The echocardiographic windows for the Doppler record in normal New Zealand white rabbits were determined. The characteristics of the different transvalvular flows and normal values for the left ventricle isovolumetric relaxation time (IVRT), the peak velocity (Vp) and ventricular ejection time (Tey) for the sigmoid valves flow, were also established. For atrioventricular flows the peak velocity for the $\mathrm{E}$ and $\mathrm{A}$ waves and the $\mathrm{E} / \mathrm{A}$ relation for the transmitral flow were registered.
\end{abstract}

Keywords: rabbit, Doppler echocardiography, transvalvular flow.

\section{INTRODUCCIÓN}

La ultrasonografía cardíaca, tanto en su modalidad bidimensional como Doppler, es el método no invasivo por excelencia a la hora de realizar la evaluación anatómica y funcional del corazón. El método se utiliza en forma rutinaria en seres humanos y especies domésticas desde hace décadas; recientemente ha adquirido importancia en los roedores (conejos, ratas y ratones) dado que la investigación médica los ha tomado como modelos experimentales ${ }^{5-7,9-17,20,22}$. De la misma manera que ocurrió con la ecocardiografía bidimensional, técnicas más sofisticadas como las distintas formas de Doppler (espectral, color y tisular) se han comenzado a utilizar como herramientas de diagnóstico en la medicina experimental ${ }^{8,21}$.

En estudios previos se han establecido protocolos de sujeción y exploración para los conejos, teniendo en cuenta su anatomía. Se ensayaron varias ventanas, rotaciones y angulaciones del transductor sobre ambos hemitórax a nivel del $3^{\circ}$ y $4^{\circ}$ espacio intercostal, y en

Recibido: 27 setiembre 2010 / Aceptado: 11 octubre 2010 *Proyecto VE-016 SECyT-UBA, 2008-2010, aprobado por el Comité Institucional de Cuidado y Uso de Animales de Experimentación, Protocolo 2007/13. el epigastrio para la exploración ecocardiográfica bidimensional, dado que la posición anatómica del corazón dentro del tórax en el conejo difiere respecto de los caninos y felinos ${ }^{2-4,19}$.

Si bien muchas de las ventanas y sus imágenes dentro de ellas son similares para la ecocardiografía bidimensional y el estudio Doppler, otras veces es necesario buscar mejores posiciones que permitan lograr una óptima alineación entre el haz de ultrasonido y la dirección del flujo a estudiar ${ }^{19}$.

Actualmente, mucha de la investigación basica cardiológica se realiza sobre el conejo neozelandés blanco por ser éste un modelo que presenta varias ventajas ${ }^{5-7}$, 9-13, 15, 16, 20. Contar con tecnicas de estudio bien establecidas y con valores de referencia normales de las distintas variables utilizadas con mayor frecuencia es fundamental cuando una especie es tomada como modelo experimental, dado que solo a partir de lo normal puede inferirse lo patológico.

Durante la etapa de rastreo bibliográfico se observó la existencia de una amplia dipersion de técnicas y valores normales de los distintos parametros Doppler en la mencionada especie y raza. Dicha variación puede atribuirse a multiples factores (habilidad de los operadores, calidad y tipo del equipamiento). Es por dicha razón que la mayoría de los laboratorios de investiga- 
ción desarrollan y utilizan sus propios valores, dado que ello permite ajustar mejor los resultados de los experimentos (téngase en cuenta que en los protocolos de cualquier estudio se incluyen los testigos simulados "sham" para comparar con el grupo experimental) ${ }^{8,21}$.

Los objetivos del presente estudio fueron establecer las mejores ventanas para el estudio de los cuatro flujos transvalvulares, estudiar la morfología de dichos flujos y determinar los valores normales para el tiempo de relajación isovolumétrico (TRIV) del ventrículo izquierdo, la velocidad pico (Vp) y tiempo de eyeccion (Tey) ventricular para las válvulas sigmoideas y para los flujos atrioventriculares la velocidad pico para las ondas E y A, tiempo de desaceleración de la onda E y relación E/A para el flujo transmitral.

\section{MATERIAL Y MÉTODOS}

Se utilizaron 63 conejos de raza neozelandés machos y hembras, sin evidencias de enfermedad cardíaca ni respiratoria preexistente, acorde al examen clínico, electrocardiograma, y radiografía torácica.

Los conejos provinieron en su totalidad del bioterio de la Subsede Veterinaria del Instituto de Fisiopatología Cardiovascular de la Universidad de Buenos Aires, desparasitados y sostenidos con una dieta de alimento balanceado para conejos suministrado ad-libitum y con pesos entre 2,5 y $3,0 \mathrm{~kg}$ al momento del estudio. Los ecocardiogramas se realizaron en el laboratorio del mencionado instituto.

Se utilizaron tres ecógrafos: Kontron, modelo Sigma Iris 440 con transductor sectorial mecánico de 7,5 MHz, Fukuda-Denshi modelo 750XT con transductor microconvexo multifrecuencia $(6,5-8 \mathrm{MHz})$ y SonoScape, modelo S8 con transductor phased array multifrecuencia (4-7 MHz) y microconvexo multifrecuencia

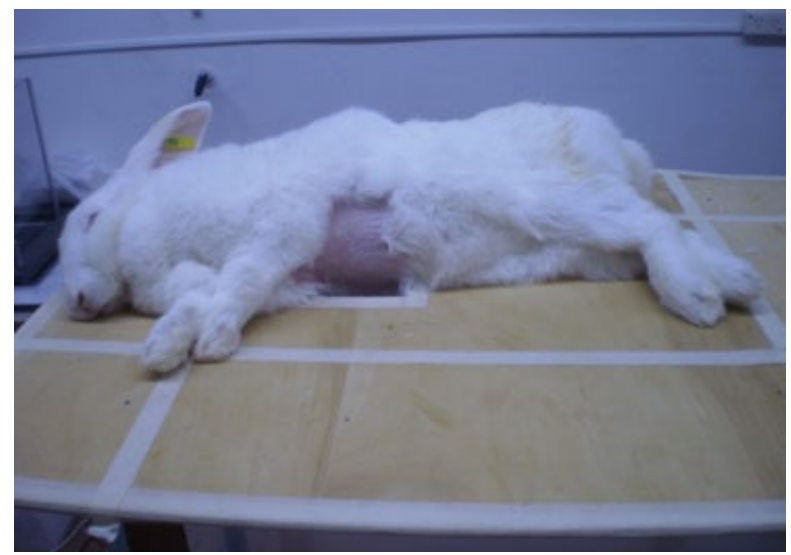

Figura 1. Conejo posicionado en decúbito lateral derecho sobre una camilla con ventana central que permite el abordaje del corazón favoreciendo el contacto cardíaco con la pared y mejorando la calidad de las imágenes.

(5-9 MHz). Como equipos periféricos se emplearon una videoimpresora marca Sony modelo UP 870MD, una videograbadora VHF, marca Philips modelo VR354 y un almacenador de video externo, marca Pinnacle.

Con el fin de minimizar los movimientos de los pacientes durante el examen y acelerar el tiempo del estudio éstos fueron sedados 5 a 10 minutos antes de realizar los estudios. Para la sedación se utilizó midazolam en dosis de $2 \mathrm{mg} / \mathrm{kg}$ por vía intramuscular ${ }^{18}$. Luego de la sedación se practicó una escrupulosa tricotomía en los sectores a explorar (ventanas), hecho de importancia pues el pelo de conejo, por sus características aislantes impide el adecuado contacto piel-transductor, resultando esto en la obtención de imágenes de baja calidad.

Los estudios ecográficos se realizaron sobre una camilla desarrollada "ad hoc" (Figura 1) y la metodología empleada fue la siguiente:
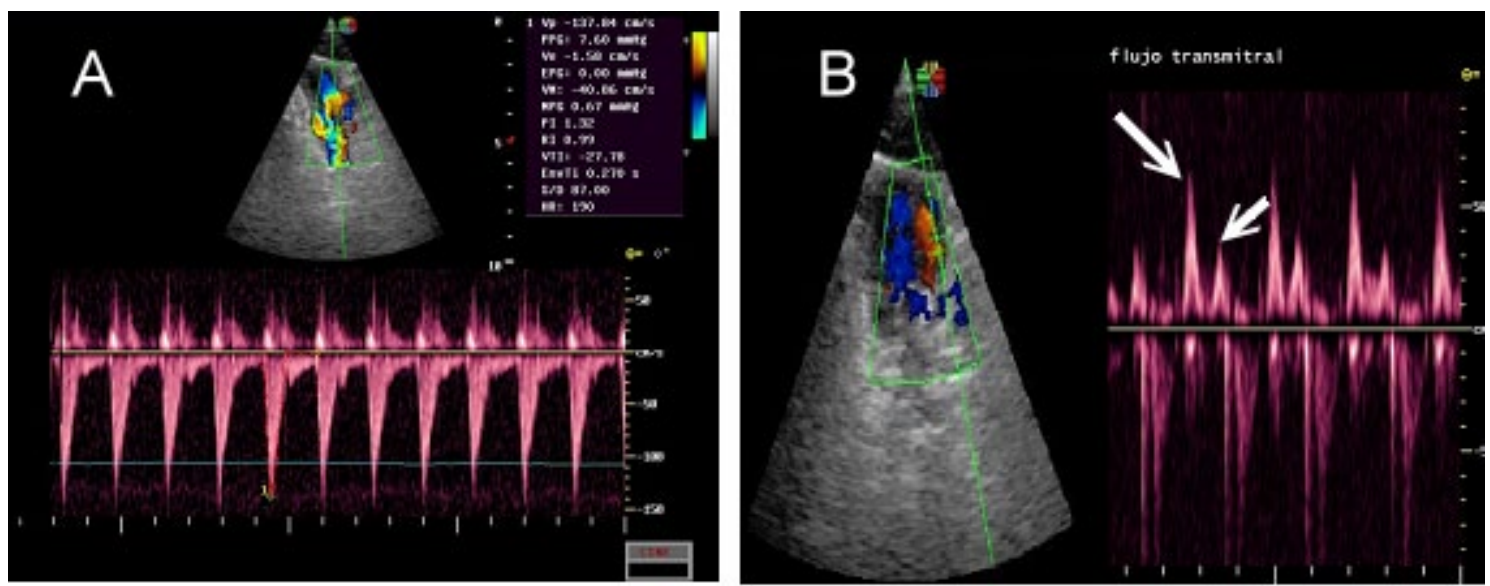

Figura 2. Flujos intracardíacos del sector izquierdo. A) Flujo transaórtico: en la imagen bidimensional color (arriba centro) se observa claramente en el medio la arteria aorta, la cual se encuentra perfectamente alineada con el haz de ultrasonido. En la imagen Doppler espectral (abajo) se observa una onda negativa, cuyo tiempo de aceleración es mucho menor que el de desaceleración, característica típica del espectro transaórtico. B) Flujo transmitral: con el volumen de muestra en el punto de máxima apertura de la válvula mitral (en la cavidad del ventrículo izquierdo) se observa un espectro bifásico positivo, donde la flecha larga indica la onda E que representa el llenado protodiastólico y la flecha corta a la onda A, correspondiente al llenado ventricular telediastólico. 


\section{Flujo transaórtico}

Se exploró con el conejo en decúbito lateral derecho desde la ventana subxifoidea. En todos los casos en los cuales se empleó esta ventana, se utilizó posición de Trendelemburg. El transductor se apoyó sobre la superficie abdominal realizando presión en sentido craneal y la marca indicadora del barrido se colocó en relación a la pared del epigastrio. La imagen bidimensional obtenida mostró la válvula y la arteria aorta en posición central, con su flujo sanguíneo perfectamente alineado con el haz de ultrasonido. El volumen de muestra, con una abertura de $2 \mathrm{~mm}$, se colocó en el centro del punto de máxima apertura de las valvas de la válvula aorta, dentro del vaso (Figura 2A).

\section{Flujo transmitral}

Se obtuvo con el conejo en decúbito lateral derecho desde la ventana subxifoidea. La posición del transductor fue semejante a la descripta para el estudio transaórtico y la marca indicadora del barrido se colocó en relación a la pared del epigastrio ligeramente rotada hacia la izquierda de la línea media. La imagen obtenida mostró la válvula mitral y sus dos valvas. El volumen de muestra se colocó en el centro del punto de máxima apertura de las valvas valvulares en la cavidad del ventrículo izquierdo (Figura 2B).

\section{Flujo transpulmonar}

La exploración del flujo transpulmonar se realizó desde dos posiciones diferentes, siempre con el paciente en decúbito lateral derecho. La primera desde la ventana paraesternal derecha, en la imagen en eje corto a nivel a la altura de la base cardíaca (Figura 3A). La segunda posición fue desde la ventana subxifoidea, girando el transductor unos $25^{\circ}$ a la derecha respecto de la posición descripta previamente para la aorta (Figura 3B). Un hallazgo relativamente constante fue la presencia de una muesca mesosistólica en el flujo transpulmonar cuando se lo observó desde la ventana subxifoidea (Figura 3B).
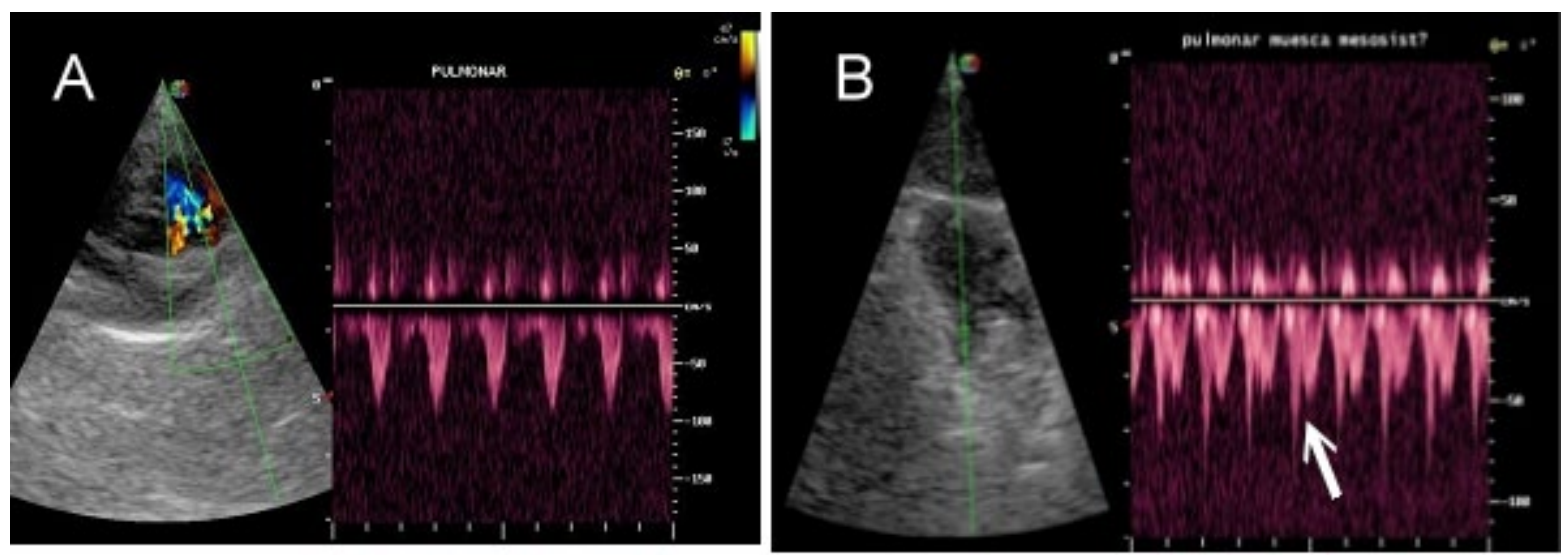

Figura 3. Flujo transpulmonar: A) Ventana paraesternal derecha, imagen en eje corto a nivel de la base cardíaca. El flujo se expresa en el Doppler espectral como una onda negativa cuyas pendientes de aceleración y desaceleración son prácticamente iguales. B) Ventana subxifoidea: se ubica esta válvula en la imagen bidimensional hacia la izquierda de la válvula aórtica, mostrando un espectro negativo frecuentemente con muesca mesosistólica (flecha).
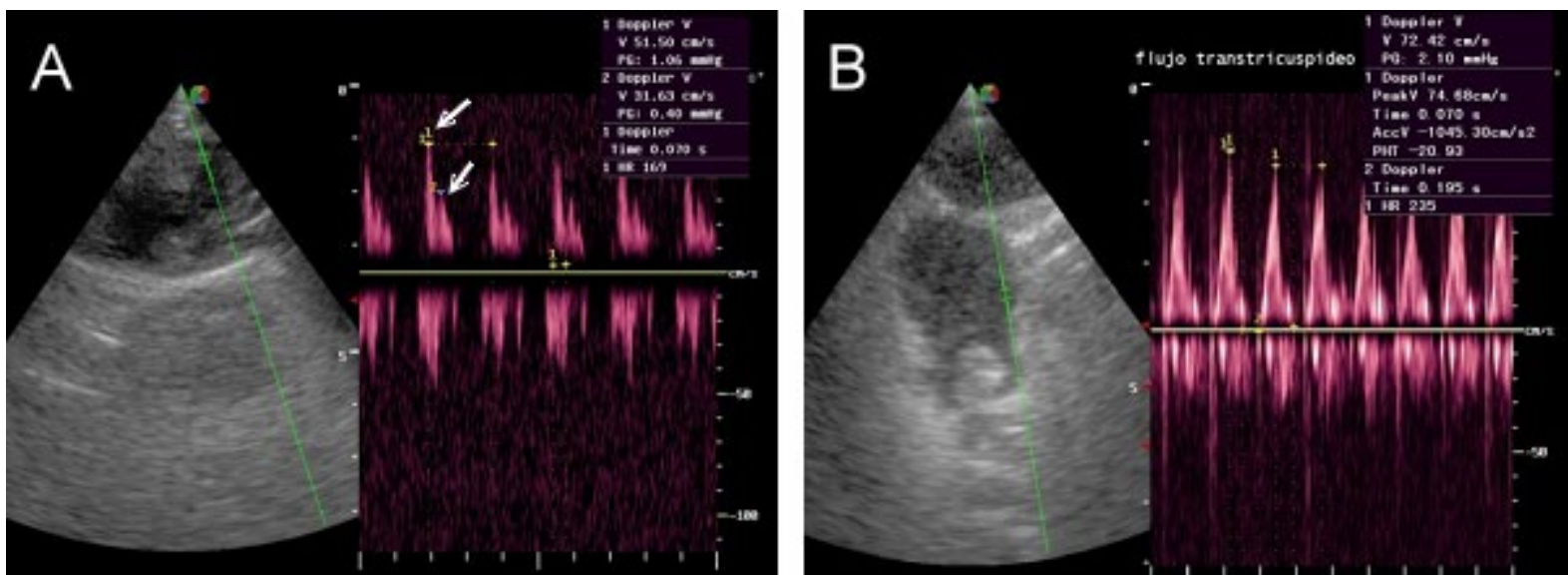

Figura 4. Flujo transtricuspídeo A) Ventana paraesternal derecha, imagen de la base cardíaca. La imagen muestra un flujo bifásico (ondas E y A) positivo, idéntico al que muestra el llenado ventricular izquierdo. B) Ventana subcostal. Cuando la frecuencia cardíaca se eleva puede aparecer un patrón monofásico debido a que las ondas E y A se superponen. 
Tabla 1. Resumen de los valores determinados.

\begin{tabular}{|c|c|c|c|c|c|c|c|c|c|c|c|c|}
\hline & \multirow[b]{2}{*}{$\begin{array}{c}\text { TRIV } \\
\mathrm{ms} \\
\mathrm{n}=40\end{array}$} & \multirow[b]{2}{*}{$\begin{array}{l}\mathrm{FC} \\
\mathrm{lpm} \\
\mathrm{n}=63\end{array}$} & \multicolumn{2}{|c|}{$\begin{array}{l}\text { V. Aórtica } \\
n=63\end{array}$} & \multicolumn{2}{|c|}{$\begin{array}{l}\text { V. Pulmonar } \\
\mathrm{n}=63\end{array}$} & \multicolumn{3}{|c|}{ V. Mitral } & \multicolumn{3}{|c|}{ V. Tricúspide } \\
\hline & & & $\begin{array}{c}\mathrm{Vp} \\
\mathrm{cm} / \mathrm{seg}\end{array}$ & $\begin{array}{l}\text { Pey } \\
\text { ms }\end{array}$ & $\begin{array}{c}\mathrm{Vp} \\
\mathrm{cm} / \mathrm{seg}\end{array}$ & $\begin{array}{l}\text { Pey } \\
\text { ms }\end{array}$ & $\begin{array}{c}\mathrm{Vp} \mathrm{E} \mathrm{cm/} \\
\text { seg } \\
\mathrm{n}=63\end{array}$ & $\begin{array}{c}\mathrm{Vp} \mathrm{A} \\
\mathrm{cm} / \mathrm{seg} \\
\mathrm{n}=63\end{array}$ & $\begin{array}{c}\text { Tdes E } \\
\mathrm{ms} \\
\mathrm{n}=56\end{array}$ & $\begin{array}{c}\mathrm{Vp} \mathrm{E} \\
\mathrm{cm} / \mathrm{seg} \\
\mathrm{n}=63\end{array}$ & $\begin{array}{c}\mathrm{VpA} \\
\mathrm{cm} / \mathrm{seg} \\
\mathrm{n}=63\end{array}$ & $\begin{array}{c}\text { Tdes E } \\
\text { ms } \\
n=17\end{array}$ \\
\hline media & 45 & 223,25 & $-102,78$ & 130,60 & $-79,45$ & 166,8 & 61,05 & 33,27 & 75 & 58,14 & 32,49 & 6 \\
\hline $\mathrm{DE}$ & 5,54 & 31,19 & $-4,298$ & 13,90 & $-5,74$ & 0,02 & 5,17 & 2,97 & 0,01 & 12,10 & 17,17 & 1 \\
\hline $\mathrm{EE}$ & 0,87 & 3,93 & $-0,537$ & 1,75 & $-0,71$ & 0,003 & 0,65 & 0,37 & 0,0013 & 1,53 & 2,16 & 0,24 \\
\hline
\end{tabular}

TRIV: tiempo de relajación isovolumétrica; FC: frecuencia cardíaca; Vp: velocidad pico; Pey: período eyectivo ventricular; Tdes: tiempo de desaceleración, DE: desvío estándar; EE: error estándar; ms: milisegundos; lpm: latidos por minuto.

\section{Flujo transtricuspídeo}

Se estudió con el paciente en decúbito lateral derecho desde la ventana paraesternal derecha en eje corto a nivel de la base cardíaca y desde la ventana subcostal izquierda. Cuando se realizó la exploración desde la ventana paraesternal derecha el volumen de muestra se colocó en el punto de máxima apertura de las valvas tratando de lograr la mayor alineación de ambos flujos (Figura 4A). Cuando la exploración se realizó desde la ventana subcostal se giró el transductor hacia la derecha lo necesario como para poder visualizar el ventrículo derecho y se colocó el volumen de muestra en el centro de la punta de la valvas en su máxima apertura, dentro del ventrículo derecho. (Figura 4B).

Las mediciones de los distintos accidentes se realizaron de la siguiente manera: TRIV: el volumen de muestra se colocó en un punto intermedio entre el flujo de salida ventricular y el de entrada, midiendo el es-

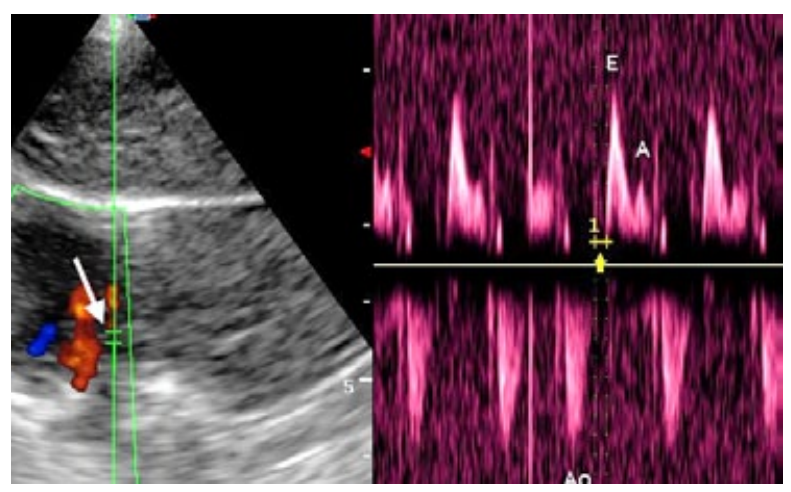

Figura 5. Forma en que se realiza la medición del tiempo de relajación isovolumétrica del ventrículo izquierdo. El analizador de volumen (flecha) se coloca en un punto medio entre el flujo de salida ventricular y el de llenado. El número 1 en el trazado Doppler muestra el punto de medición. Vp: la medición se realiza en el punto máximo (negativo o positivo) que alcanza la onda de flujo. Tey: el tiempo de eyección ventricular se mide desde el principio hasta el final de la correspondiente onda de flujo. Tdes: se mide el tiempo entre el punto de la $\mathrm{Vp}$ hasta el final de la onda sobre la línea de base. En todos los casos se empleó el filtro de pared del menor valor posible con el objeto de poder realizar adecuadamente las mediciones sobre la línea de base. pacio de tiempo que media entre el fin de la onda de vaciado ventricular (aórtica o pulmonar) y el principio de la de llenado ventricular (onda E) (Figura 5).

El procesamiento estadístico consistió en el cálculo de la media aritmética, el desvío estándar y el error estándar. Los mencionados valores se obtuvieron mediante un software (Statistix 8). Cabe aclarar que el valor final tomado como normal, fue el promedio de tres mediciones recogidas de ecotomogramas diferentes.

\section{RESULTADOS Y DISCUSIÓN}

El equipamiento utilizado para la captura Doppler fue en todos los casos adecuado, en la medida en que su calibrado fue regulado atendiendo, fundamentalmente, a optimizar su frame rate debido a la alta frecuencia del corazón del conejo y la consiguiente superposición de imágenes. Ello se logra fundamentalmente cerrando lo máximo posible el ángulo de exploración y disminuyendo al mínimo la profundidad de estudio.

Debido a las características propias de la especie, es necesario contar con cierto grado de sujeción para facilitar la realización de los estudios. Se eligió el protocolo con midazolam por su rápida acción y corto efecto (el necesario para realizar el estudio) y mínimos o nulos efectos sobre el sistema cardiovascular como lo demuestran las escasas variaciones de la frecuencia cardíaca (Tabla 1).

Respecto de las ventanas de exploración es importante destacar la utilidad de las ventanas subxifoidea y subcostal izquierda. Tanto una como otra permiten obtener buenas imágenes bidimensionales en eje largo y una perfecta alineación con los flujos transvalvulares

$\mathrm{Al}$ respecto, puede mencionarse que la ventana subcostal izquierda permite un óptimo registro de los flujos transaórtico, transmitral y transpulmonar, pero no posibilita una buena alineación para la captura del flujo transtricuspídeo. El flujo de esta válvula se logra mejor a través de la ventana subxifoidea.

Un hecho importante de resaltar es la aparición de una muesca mesosistólica cuando se explora el flujo transpulmonar desde la ventana subxifoidea. Dicha muesca es considerada, en el resto de las especies domésticas y en seres humanos, como un signo característico de hipertensión pulmonar. Sin embargo, de 
acuerdo a nuestros resultados esto sería, en esta posición, un hecho normal en el conejo ${ }^{1}$.

Las ventanas tradicionales no fueron en general adecuadas para el registro de los flujos intracardíacos, debido a la imposibilidad de obtener una buena alineación del ultrasonido con el flujo a explorar, indispensable para poder realizar una correcta medición.

En general, todos los flujos transvalvulares en el conejo respetan lo que se ha descripto en la mayoría de los mamíferos. Las características particulares son las siguientes: el flujo transaórtico (vaciado ventricular izquierdo) se caracteriza por formar una onda negativa desde la ventana subxifoidea dado que el flujo se aleja del transductor. La forma semeja un triángulo rectángulo debido al llamativamente corto tiempo de aceleración y relativamente largo tiempo de desaceleración (Figura 2B).

El flujo transmitral (llenado ventricular izquierdo) está representado por dos ondas, la primera (onda E) representa el llenado ventricular rápido (protodiastólico). Tiene mayor velocidad que la segunda onda de llenado e igualdad en los tiempos de aceleración y desaceleración. El segundo componente es la denominada onda A, que representa el llenado ventricular tardío (telediastólico o presistólico) producido a expensas de la contracción atrial.

El flujo transpulmonar (vaciado ventricular derecho) captado desde cualquiera de las dos ventanas estudiadas se manifiesta por una onda negativa dado que el movimiento sanguíneo se aleja del transductor. En general la onda presenta menor velocidad que la del flujo transaórtico (Tabla 1). Se diferencia de éste dado que, tanto el tiempo de aceleración como el de desaceleración son casi idénticos, lo cual otorga a la onda una característica forma de triángulo isósceles. Como se mencionó previamente en la ventana subxifoidea aparece una disminución pasajera de la velocidad de la onda de flujo transpulmonar, conocida como "muesca mesosistólica".

El flujo transtricuspídeo (llenado ventricular derecho) normal en el conejo es esencialmente similar al de llenado ventricular izquierdo, compuesto por dos ondas (E y A), como ocurre en la mayoría de las especies. Debido a la imposibilidad de una adecuada alineación, en general las ondas suelen tener bastante dispersión espectral.

Los valores obtenidos se encuentran resumidos en la Tabla 1 para facilitar su lectura. Los valores de velocidad obtenidos en el presente estudio fueron más elevados que los publicados por otros autores ${ }^{7}$. Cabe aclarar, que en la exploración Doppler la falta de alineación adecuada subestima los flujos, de tal forma que los valores obtenidos por esta técnica siempre subestiman el verdadero valor y nunca lo sobrestiman, razón por la cual siempre debe contabilizarse el valor más alto registrado.

Se concluye que la ecocardiografía Doppler (espectral y color) es una técnica diagnóstica de fácil realización, mínima invasividad y corto tiempo de ejecución que permite evaluar en forma cuali-cuantitativa los flujos intracardíacos en conejos. Las ventanas de elección para la captura de todos los flujos intracardíacos son la subxifoidea y la subcostal izquierda pues permiten una óptima alineación del ultrasonido con los flujos a explorar. La presencia de la muesca mesosistólica observada en el flujo transpulmonar, cuando se lo explora desde la ventana subxifoidea, parece ser un evento normal en el conejo.

\section{REFERENCIAS}

1. Adaniya M. 2005. Valvulopatías. En: Ecocardiografía para la toma de decisiones clínicas (Piñeiro D Ed), Panamericana, Buenos Aires, 629-633.

2. Chiaramonte P, Pidal G, D'Anna E, Lightowler C. 2009. Ultrasonografía cardíaca en conejos, descripción de ecotomogramas bidimensionales básicos. Memorias $6^{\text {tas }}$ Jorn Intern Vet Práct. On line: www.cvpba.org/assets/pdf/ revista_43.pdf, Mar del Plata, Argentina

3. Chiaramonte P, Pidal G, Lightowler C. 2009. Parámetros ecocardiográficos para la evaluación de la función sistólica en conejos. Anales Jorn Hosp Fac Cs Vet UBA, Buenos Aires, p. 32.

4. Chiaramonte P, Pidal G, Lightowler C. 2010. Ecocardiografía bidimensional en conejos neozelandeses blancos normales. Rev InVet 12: 33-42.

5. Cuniberti L, Stutzbach P, Guevara E, Yannarelli G, Laguens R, Favaloro R. 2006. Development of mild aortic valve stenosis in a rabbit model of hypertension. $\mathrm{J} \mathrm{Am}$ Col Cardiol 47: 2303-2309.

6. Donato M, Morales C, Bagnarelli A, Scapín O, Gelpi R. 1999. Adenosina exógena y disfunción post-isquémica ("miocardio atontado") en el corazón aislado de conejo. Medicina (Buenos Aires) 59: 339-347.

7. Downey J, Miura T, Eddy L. 1987. Xanthine oxidase is not a source of free radicals in the ischemic rabbit heart. $J$ Mol Cell Cardiol 19: 1053-1060.

8. Fontes-Sousa A, Brás-Silva C, Moura C, Areias J, Leite-Moreira A. 2006. M-mode and Doppler echocardiographic reference values for male New Zeland White rabbits. Am J Res 67: 1725-1729.

9. Gelpi R, Morales C, Rodriguez M, Bagnarelli A, Hita A, Scapin O. 1998. Efectos del enalaprilato sobre la disfunción post-isquémica sistólica y diastólica ("miocardio atontado") en el corazón aislado de conejo. Medicina (Buenos Aires) 58: 22-28.

10. González G, Mangas F, Depetris A, Monroy S, Donato M, Morales C, Gelpi R. 2003. Comportamiento diastólico al comienzo de la reperfusión en el miocardio atontado de conejo. Medicina (Buenos-Aires) 63: 403-409.

11. Gonzalez G, Mangas F, Palleiro J, Rodriguez M, Depetris A, Gelpi R, Morales C. 2004. Efectos de la administración temprana de losartan sobre el remodelamiento ventricular en conejos con infarto de miocardio experimental. Medicina (Buenos Aires) 64: 25-29.

12. González G, Seropian I, Krieger L, Palleiro J, López Verrilli M, Gironacci M, Cavallero S, Tomasi V, Gelpi R, Morales C. 2009. Effect of early vs late AT1 receptor 
blockade with losartan on post-myocardial infarction ventricular remodeling in rabbits. Am J Physiol Heart Circ Physiol 297: 375-386.

13. Marian A. 1999. A transgenic rabbit model for human hypertrophic cardiomyopathy. J Clin Invest 104: 1684-1692.

14. Maxwell M, Hearse D, Yellon D. 1987. Species variation in the coronary collateral circulation during regional myocardial ischaemia: a critical determinant of the rate of evolution and extent of myocardial infarction. Cardiovasc Res 21: 737-746.

15. Morales C, Rodriguez M, Scapin O, Gelpi R. 1998. Comparison of the effects of ACE inhibition with those of angiotensin II receptor antagonism on systolic and diastolic myocardial stunning in isolated rabbit heart. $J \mathrm{Mol}$ Cell Biochem 186:117-121.

16. Morales C, Rodriguez M, Gonzalez G, Matoso M, Bertolasi C, Gelpi R. 2001. Cronodinamia del infarto de miocardio en el corazón de conejo. Medicina (Buenos Aires) 61: 830-836.

17. Mosca S, Gelpi R, Milei J, Fernandez Alonso G, Cingolani H. 1998. Is stunning prevented by ischemic preconditioning?. J Mol Cell Biochem 186: 123-129.
18. Muir W, Hubbell J, Bednarski R. 2007. Manual de anestesia veterinaria, $4^{\circ}$ ed, Elsevier-Mosby, Zaragoza, p. 474.

19. Pidal G, Buzzano O, Chiaramonte P, Graziano A, Lightowler C. 2008. Ventanas ecotomográficas para el corazón del conejo. Anales $1{ }^{\circ}$ Congr Vet Hosp Corrientes, Argentina, tomo 5, p. 105.

20. Plehn J, Foster E, Grice M. 1993. Echocardiographic assessment of LV mass in rabbits: models of pressure and overload hypertrophy. Am J Physiol Heart Circ Physiol 265: H2066-H2072.

21. Stypmann J, Engelen M, Breithardt P, Rothenburger M, Breithardt O, Breithardt B, Eckardt L, Cordula P. 2007. Doppler echocardiography and tissue doppler imaging in the healthy rabbit: ifferences of cardiac function during awake and anesthetized examination. Int $J$ Cardiol 115: 164-170.

22. Verdouw P, van den Doel M, Zeeuw S, Duncker D. 1998. Animal models in the study of myocardial ischaemia and ischaemic syndromes. Cardiovasc Res 39: 121-135. 